\title{
Role of rice stripe virus NSvc4 in cell-to-cell movement and symptom development in Nicotiana benthamiana
}

\section{Yi Xu and Xueping Zhou*}

State Key Laboratory of Rice Biology, Institute of Biotechnology, Zhejiang University, Hangzhou, China

\section{Edited by:}

Aiming Wang, Agriculture and

Agri-Food Canada, Canada

\section{Reviewed by:}

Taiyun Wei, Fujian Agriculture and

Forestry University, China

Weimin Li, Chinese Academy of

Agricultural Sciences, China

Andrew O. Jackson, University of

California, Berkeley, USA

${ }^{*}$ Correspondence:

Xueping Zhou, State Key Laboratory of Rice Biology, Institute of

Biotechnology, Zhejiang University, Hangzhou, Zhejiang 310058, China.

e-mail: zzhou@zju.edu.cn
Our previous work has demonstrated that the NSvc4 protein of Rice stripe virus (RSV) functions as a cell-to-cell movement protein. However, the mechanisms whereby RSV traffics through plasmodesmata (PD) are unknown. Here we provide evidence that the NSvc4 moves on the actin filament and endoplasmic reticulum network, but not microtubules, to reach cell wall PD. Disruption of cytoskeleton using different inhibitors altered NSvc4 localization to PD, thus impeding RSV infection of Nicotiana benthamiana. Sequence analyses and deletion mutagenesis experiment revealed that the $\mathrm{N}$-terminal 125 amino acids (AAs) of the NSvc4 determine PD targeting and that a transmembrane domain spanning AAs 106-125 is critical for PD localization. We also found that the NSvc4 protein can localize to chloroplasts in infected cells. Analyses using deletion mutants revealed that the $\mathrm{N}$-terminal 73 AAs are essential for chloroplast localization. Furthermore, expression of NSvc4 from a Potato virus $X$ (PVX) vector resulted in more severe disease symptoms than PVX alone in systemically infected $N$. benthamiana leaves. Expression of NSvc4 in Spodoptera frugiperda 9 cells did not elicit tubule formation, but instead resulted in punctate foci at the plasma membrane. These findings shed new light on our understanding of the movement mechanisms whereby RSV infects host plants.

Keywords: rice stripe virus, movement, chloroplast, tubules

\section{INTRODUCTION}

Rice stripe disease is the most devastating viral disease of rice in China, Japan, and Korea (Wei et al., 2009). The causal agent, Rice stripe virus (RSV), is the type member of the Tenuivirus genus and the viral genome consists of four single-stranded RNA segments (RNAs 1, 2, 3, and 4; Hibino, 1996). RNA 1 is negative-sense and encodes a putative RNA-dependent RNA polymerase. RNAs 2, 3, and 4 are ambisense, and each of which encodes two open reading frames (ORFs) with one on viral RNA (vRNA) and another on viral complementary RNA (vcRNA). RSV vRNA 2 encodes a membrane-associated protein that reportedly is an RNA silencing suppressor and interacts with SGS3 (Du et al., 2011). The vcRNA 2 encodes a glycoprotein with unidentified functions (Zhao et al., 2012). The vRNA 3 and vcRNA 3 encode a gene silencing suppressor and a nucleocapsid (NC) protein, respectively (Hibino, 1996; Xiong et al., 2009). RSV vRNA 4 encodes a disease-specific protein that accumulates in both infected plant and insect cells (Toriyama, 1986). The protein encoded by vcRNA 4 was identified as the RSV movement protein (MP; Xiong et al., 2008). RSV is transovarially transmitted by small brown planthopper (SBPH), Laodelphax striatellus, in a circulative-propagative manner (Falk and Tsai, 1998; Li et al., 2011). After RSV infection, rice plants often show chlorotic stripes in the newly expanded leaves, and the stripes progress into pale streaks in infected plant leaves. Because of global environment changes and the extensive increases in distribution of the transmission vector (L. striatellus) in the south and southeastern parts of China, RSV has caused significant losses in rice production in the past decade.
To infect a host plant successfully, viruses must overcome two obstacles; they must be capable of replicating in host cells and moving between cells and then be able to move systemically throughout the plant via the vasculature. To carry out these functions, viruses encode MPs that often interact with viral genomic (g) RNAs to form ribonucleoprotein complexes that mediate intra- and intercellular movement. At the plasmodesmata (PD), the MPs modify PD size exclusion limits to enable transit of the ribonucleoprotein complexes to adjacent cells. In some examples, the viral MPs form tubules that penetrate through the PD and serve as conduits for whole virus cell-to-cell transport. It has been shown that viruses often co-opt plant cellular processes to carry out specific functions required for infection (Scholthof, 2005; Shen et al., 2011). In addition to host factors that interact directly with viral MPs (Paape et al., 2006; Shimizu et al., 2009), host cytoskeleton, and endoplasmic reticulum (ER) networks also play critical roles in virus movement in hosts (Ashby et al., 2006; Harries et al., 2010). For example, both microtubules and microfilaments have been implicated in supporting cell-to-cell movement of Tobacco mosaic virus (TMV) in Nicotiana benthamiana (Brandner et al., 2008; Harries et al., 2009b, 2010). The MP of Abutilon mosaic virus (AbMV) is known to have an anchor domain that allows the MP to localize to the ER (Aberl et al., 2002). Association of viral MPs with the secretory pathway was also reported for viruses whose MPs form tubules. For example in cells infected with Cowpea mosaic virus or Cauliflower mosaic virus, tubule formation was independent of microtubules or microfilaments, but tubule formation required a functional secretory pathway (Huang 
et al., 2000; Pouwels et al., 2002). Trafficking of P3N-PIPO and CI of Turnip mosaic virus (TuMV) to PD has also been shown to be dependent on the host secretory pathway (Wei et al., 2010b). Interestingly, in Grapevine fanleaf virus infected cells both the secretory pathway and the cytoskeleton networks were reported to be involved in tubule formation and in intra-cellular targeting of virions (Laporte et al., 2003). Thus, plant virus may utilize the host cytoskeleton, the ER network, or both for PD targeting. Genomes of plant viruses are small and each virus encodes only a few proteins. Consequently, virus-encoded proteins are often multi-functional proteins. For example, the coat protein of Turnip crinkle virus (TCV) not only functions in movement between cells and in virion assembly, but also functions as a suppressor of gene silencing (Qu et al., 2003; Cao et al., 2010). Viral MPs also have varied functions: $\mathrm{BC} 1$ of AbMV accumulates preferentially at the cell periphery or around the nucleus in plant cells, and hence may participate in distinct functions (Zhang et al., 2001, 2002). The Barley stripe mosaic virus-encoded triple-gene block (TGB) 1 protein has similar localization patterns, and TGB2 can localize to both ER membranes and chloroplasts, indicating it also has distinct functions (Torrance et al., 2006; Lim et al., 2009). Several other viral MPs have been reported to accumulate in chloroplasts and are considered to have important roles in virus replication, viral transport, or symptom development. For example, mutation of the chloroplast-targeting signal in the Alternanthera mosaic virus (AltMV) TGB3 impaired the virus cellto-cell movement and eliminated the long distance movement of the virus (Lim et al., 2010). A number of biochemical and subcellular localization activities are associated with the TGB proteins of other flexiviruses, including intra-cellular targeting, gene silencing activities, and host membrane remodeling (Verchot-Lubicz et al., 2010; Tilsner et al., 2012). The $66 \mathrm{~K}$ protein of Turnip yellow mosaic virus (TYMV) was reported to localize to virus-induced chloroplastic membrane vesicles, which are thought to function as TYMV RNA replication factories (Prod'homme et al., 2003). The TuMV $6 \mathrm{~K}$ also has been shown to target chloroplasts to result in aggregation and elicitation of membrane invaginations (Wei et al., 2010a). Former work demonstrated that NSvc4 rely on the early secretory pathway and actin-myosin VIII motility system for plasmodesmatal localization and could induce foliar necrosis from a TMV-NSvc4 hybrid vector (Yuan et al., 2011; Zhang et al., 2012). Here we present new evidence indicating that NSvc4 exerts its movement functions by trafficking on actin filaments and ER networks to reach the PD and shown that the N-terminal 125 amino acids (AAs) determine the PD localization. We also demonstrate that the NSvc4 protein targets chloroplasts in infected cells and is a symptom determinant in plant.

\section{MATERIALS AND METHODS PLASMIDS CONSTRUCTIONS}

The full length ORFs of NSvc4 protein and the N- and C-terminal deletion mutants were amplified from pBin438-NSvc4 (Xiong et al., 2008) using the Phusion High-Fidelity DNA polymerase (New England Biolabs, Ipswich, USA). The NSvc4 deletion mutant (lacking AAs 106-125) was first amplified via an overlap PCR method with the primers MP-Fol and MP-Rol (See Table A1 in Appendix for all the primers used in this study). The resulting
PCR fragments were ligated individually into the pCHF3-eGFP plasmid and used for agroinfiltration into N. benthamiana (Xiong et al., 2008). To construct Potato virus X (PVX) NSvc4 expression vectors, full length and deletion mutants of NSvc4 were PCR amplified with primers containing ClaI and SalI restriction sites. The PCR fragments were cloned individually into the pGEMTeasy vector. After digestion using the ClaI and SalI enzymes, the resulting fragments were ligated individually to the PVX pgR107 vector (provided by Dr D. C. Baulcombe, Sainsbury Laboratory, John Innes Centre, Norwich Research Park, Norwich, UK). All the plasmids were verified by DNA sequencing before further use.

\section{PLANT INOCULATION AND CONFOCAL MICROSCOPY}

$N$. benthamiana plants were grown in a growth chamber set at $25 \pm 1^{\circ} \mathrm{C}$ and $16 \mathrm{~h}$ light and $8 \mathrm{~h}$ dark conditions. RSV infectivity trials were carried out by rub-inoculating leaves with crude extracts from RSV-infected $O$. sativa leaves ground in phosphate buffer $(0.2 \mathrm{M})$. After a 12 -h incubation in the dark, the plant were transferred to a culture room set at $25 \pm 1^{\circ} \mathrm{C}, 80 \%$ relative humidity, and $16 \mathrm{~h}$ light and $8 \mathrm{~h}$ dark cycle. Local and systemic leaf infections were evaluated at 3, 7, and 10 days post inoculation by RT-RCR (data not shown). Leaves of 4-week-old plants were infiltrated with Agrobacterium tumefaciens (strain GV3101) harboring either the full length NSvc4 sequence or one of the mutant NSvc4 plasmids using needleless syringes as described previously (Batoko et al., 2000). Leaf tissue was harvested at $48 \mathrm{~h}$ post agro-infiltration and examined for GFP fluorescence under a Leica TCS SP5 confocal microscope equipped with a $20 \times$ objective lens. Conditions set to excite GFP and monitor the emission were as described by Brandizzi et al. (2002). Chloroplast autofluorescence was detected using a $670-\mathrm{nm}$ emission filter according to the manufacturer's instructions. Confocal images were processed using the LCS Lite Leica software.

\section{INHIBITOR TREATMENTS}

Latrunculin B (LatB), oryzalin, and brefeldin A (BFA) were purchased from Sigma-Aldrich (St. Louis, USA) and dissolved in dimethyl sulfoxide (DMSO) to make stock solutions at $10 \mathrm{mM}$, $2 \mathrm{mM}$, and $200 \mu \mathrm{g} / \mathrm{ml}$, respectively. Immediately prior to use, the stocks were diluted to $5 \mu \mathrm{M}$ LatB, $50 \mu \mathrm{M}$ oryzalin, and $50 \mu \mathrm{g} / \mathrm{ml}$ BFA using double-distilled water $\left(\mathrm{ddH}_{2} \mathrm{O}\right)$. Three hours before agroinfiltration, diluted LatB, oryzalin, or BFA solutions were infiltrated into $N$. benthamiana leaves using needleless syringes as described (Harries et al., 2009a). Diluted DMSO (1:1000 in $\mathrm{ddH}_{2} \mathrm{O}$ ) was infiltrated into $N$. benthamiana leaves and used as a control. The MAN1-RFP (from soybean, which is known to localize to cis-Golgi) was used to monitoring BFA in function in our system (data not shown).

For virus inoculation assays, leaves of six-to-eight leaf stage $N$. benthamiana were rub-inoculated with $5 \mu \mathrm{M}$ LatB, $50 \mu \mathrm{M}$ oryzalin, or diluted DMSO $\left(1: 1000\right.$ in $\left.\mathrm{ddH}_{2} \mathrm{O}\right)$. One day after the chemical treatments, the leaves were rub-inoculated as described previously with crude extracts prepared from RSV-infected $O$. sativa leaves (Xiong et al., 2008). After $12 \mathrm{~h}$ incubation in the dark, the plants were transferred to a culture room set at $25 \pm 1{ }^{\circ} \mathrm{C}, 80 \%$ relative humidity, and $16 \mathrm{~h}$ light (5000 lux) and $8 \mathrm{~h}$ dark. 


\section{IMMUNOCYTOCHEMISTRY AND ELECTRON MICROSCOPY}

Small tissues (approximately $1 \mathrm{~mm}$ wide and $3 \mathrm{~mm}$ long) were excised from $N$. benthamiana leaves agroinfiltrated with the bacteria harboring the pgR107 or pgR107-NSvc4 vectors. Harvested tissues were fixed with $50 \mathrm{mM}$ phosphate-buffered saline (PBS), $\mathrm{pH} 6.8$, containing $1 \%$ glutaraldehyde and $2 \%$ formaldehyde for $3 \mathrm{~h}$ at $4^{\circ} \mathrm{C}$. After dehydration in a graded series of ethanol $(30,50$, 70,90 , and $100 \%$ ), the fixed samples were embedded in Lowicryl $\mathrm{K} 4 \mathrm{M}$ resin as described previously (Xiong et al., 2008).

\section{CONSTRUCTION OF BACULOVIRUS PLASMIDS AND TRANSFECTION OF Sf-9 CELLS}

The full length NSvc4 sequence was PCR amplified from the pgR107-NSvc4 using the primers MP-(BamH1)-F and MP-(Sal1)$\mathrm{R}$. The amplified fragments were digested with the BamHI and SalI restriction enzymes, and then inserted between the BamHI and SalI sites within the pFastBacHTGFPT transfection vector (kindly supplied by Chuanxi Zhang, Zhejiang University, Zhejiang, China) under the control of the polh promoter. The recombinant plasmid pFastBacHTGFPT-NSvc4 was transformed into E. coli DH1OBac as instructed (Invitrogen, Carlsbad, USA). After transformation, the gene cassette from the recombinant plasmid was transferred to the bacmid genome by site-specific transposition and the recombinant bacmid DNA was then isolated following the manufacturer's instructions.

Recombinant bacmid DNA was transfected into $1.0 \times 10^{6}$ cells Spodoptera frugiperda 9 (Sf-9) cell using Cellfectin Reagent (Invitrogen, Carlsbad, USA), and transfected cells were incubated at $27^{\circ} \mathrm{C}$ for $72 \mathrm{~h}$. Supernatant of culture medium TNM-FH (SigmaAldrich, USA) was collected from the transfected Sf- 9 cell cultures and transferred to fresh Sf-9 cells followed by an additional $48-72 \mathrm{~h}$ incubation period before confocal microscopy observations.

\section{COMPUTATIONAL ANALYSES OF THE NSvc4 PROTEIN}

The transmembrane helices of NSVc4 were predicted using the Membrane Protein Explorer (MPEx, version 3.2 ${ }^{1}$ ) and the $\Delta G$ prediction server $^{2}$ (Hessa et al., 2007; Snider et al., 2009). The $\Delta G$ server provides predictions of the corresponding apparent free

${ }^{1}$ http://blanco.biomol.uci.edu/mpex/

${ }^{2}$ http://dgpred.cbr.su.se/index.php?p=home energy differences. In principle, a negative $\Delta G$ value by the Sec translocon predicts that a protein sequence has a TM helix and that proteins can integrate into membranes.

\section{RESULTS}

\section{ACTIN FILAMENTS AND GOLGI APPARATUS DISRUPTION ABOLISH PD LOCALIZATION OF NSvc4 AND DELAY RSV SYSTEMIC INFECTIONS IN $\boldsymbol{N}$. BENTHAMIANA}

To investigate roles of the cytoskeleton and secretory membranes in NSvc4 intra-cellular transport and PD targeting, three pharmacological inhibitors were applied to $N$. benthamiana leaves before agroinfiltration-mediated transient expression of NSvc4eGFP. LatB and oryzalin treatments were used to disrupt actin filaments and microtubules, as previously reported (Harries et al., 2009a; Yuan et al., 2011). Expression of NSvc4-eGFP under control of the PVX pgR107-NSvc4 vector in the DMSO (control) treated $N$. benthamiana leaves yielded punctate spots at the PD (Figure 1A). When NSvc4-eGFP was expressed in LatB treated $N$. benthamiana leaves, the number of punctate spots at the PD were clearly reduced, and fluorescence was more generally dispersed throughout the cell (Figure 1B), indicating that a functional actin cytoskeleton is important for targeting NSvc4-eGFP to punctate foci at the PDs. When the NSvc4-eGFP was expressed in the oryzalin treated $N$. benthamiana leaves, abundant punctate spots similar to those noted in the DMSO controls were evident at the PD (Figure 1C). These experiments indicate that depolymerizing microtubules does not have obvious interference on formation of punctate spot at the PD. BFA is known to interfere with the ER/Golgi secretory pathway by inhibiting COPI vesicle production (Tse et al., 2006). We therefore treated N. benthamiana leaves with BFA and noted the NSvc4-eGFP fluorescence was more generally distributed in the cytosol than in the DMSO treated controls and that the localization at the PD and the peripheral membranes was greated reduced (Figures 2A,B). These results thus suggest that an intact Golgi secretory system has a substantial positive effect on PD targeting of NSv4-eGFP.

In order to determine whether the pharmacological affects were correlated with RSV infection, we inoculated N. benthamiana leaves with extracts from RSV-infected rice. We had noted earlier (Xiong et al., 2008) that RSV results in systemic infections in $N$. benthamiana after mechanical inoculation. Therefore to test
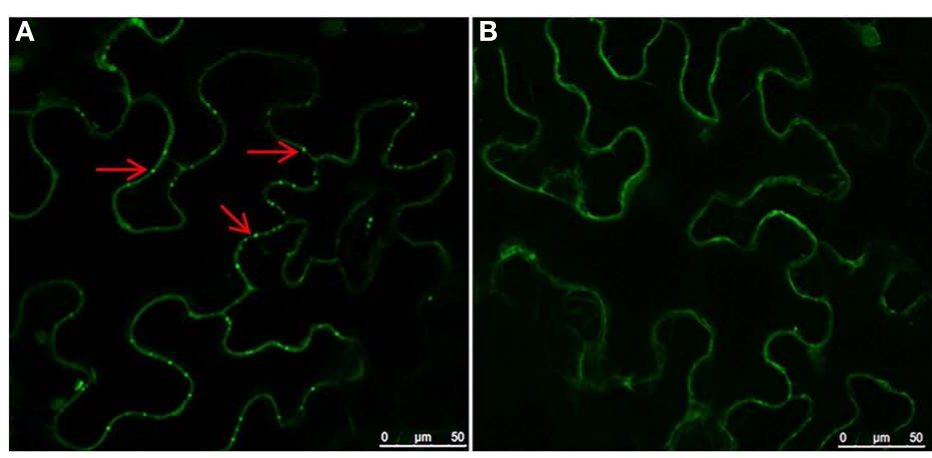

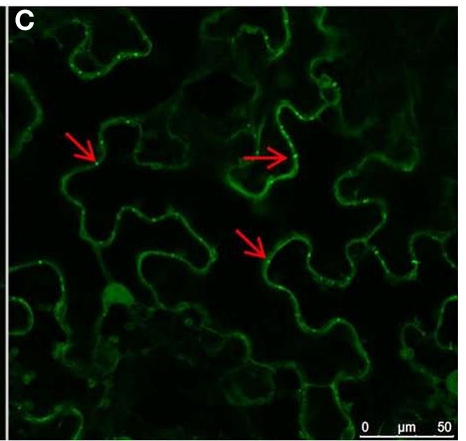

FIGURE 1 | Role of actin filaments in PD localization of NSvc4. N. benthamiana leaves were first infiltrated with DMSO (A), LatB (B), or oryzalin (C). Three hours later, the leaves were agroinfiltrated with bacteria harboring the NSvc4-eGFP vector. Infiltrated leaves were sampled at $48 \mathrm{~h}$ after agro-infiltration and subjected to examination under the confocal microscopy. Arrows indicate PD localization of fusion proteins. 

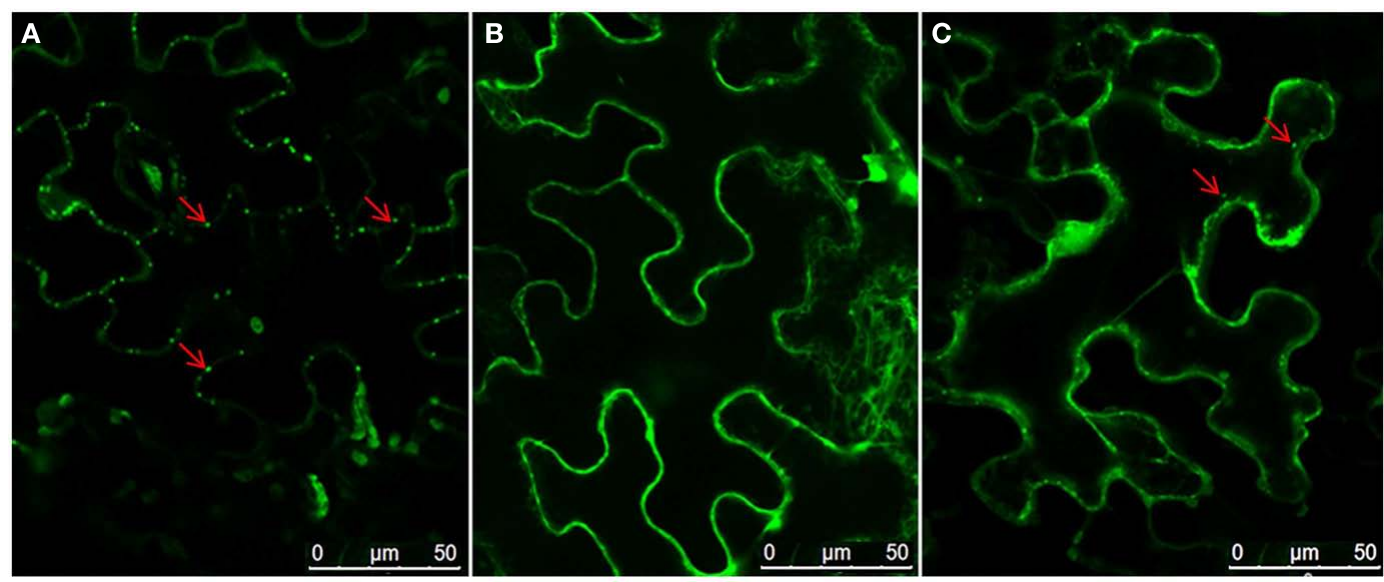

FIGURE 2 | Role of the ER-Golgi secretion pathway in PD localization of NSvc4. N. benthamiana leaves were infiltrated with DMSO (A) or BFA (B). After $3 \mathrm{~h}$, the leaves were agroinfiltrated for expression of NSvc4-eGFP. The leaf shown in (C) was agroinfiltrated with deletion mutant of NSvc4 (NSvc4 ${ }_{106-125}-\mathrm{eGFP}$ ). Arrows indicate the localization of fusion protein. the effects of the DMSO, LatB, and oryzalin treatments on RSV infection, we inoculated RSV from infected rice extracted to $N$. benthamiana leaves 1 day after application of the drug treatments. The results show that disruption of actin filaments using LatB strongly inhibits systemic infection of RSV, whereas oryzalin treatments were similar to those of DMSO on RSV systemic infection in N. benthamiana (Table 1).

\section{COMPUTATIONAL ANALYSIS OF NSvc4 AND DOMAINS RESPONSIBLE FOR NSvC4 PD LOCALIZATION}

Using the Membrane Protein Explorer program, AAs spanning positions 106-125 of the NSvc4 protein have properties of a transmembrane domain (Figure 3). To confirm this prediction, we deleted AAs 106-125 from the NSvc4 to create NSvc4 ${ }_{\Delta 106-125^{-}}$ eGFP, and expressed the mutant transiently from the PVX vector in the epidermal cells of $N$. benthamiana leaves via agro-infiltration. Confocal microscopy observations revealed that PD localization by the deletion mutant was substantially reduced compared to the DMSO controls. Only a few apparently intact foci were evident and most of the fluorescence was diffuse and appeared to be associated with the cytosol (Figure 2C). These observations provide evidence suggesting that the predicted transmembrane region (AAs 106-125) in the NSvc4 protein provides an important anchor domain that is required for NSvc4 trafficking on the endomembrane network.

To determine the domain responsible for NSvc4 PD localization, a series of NSvc4 deletion mutants were constructed, inserted into the pCHF3 vector and expressed transiently by agroinfiltration into $N$. benthamiana leaf cells. The fluorescence patterns in cells at $48 \mathrm{~h}$ after infiltration revealed that the NSvc4 ${ }_{1-54^{-}}$ eGFP, NSVc4 ${ }_{1-73-e G F P, ~ N S v c 4}{ }_{1-106}$-eGFP, and NSvc4 ${ }_{\Delta 125-286^{-}}$ eGFP mutant derivatives each elicited GFP expression patterns similar to those produced by pCHF3-eGFP, the GFP control vector (Figure 4). However, fluorescence from the NSvc4 $1-125-\mathrm{eGFP}$ deletion mutant protein accumulated in punctate foci at the PD that appeared to be similar to the fluorescence elicited in cells
Table 1 | Effect of different inhibitors on RSV infection in $N$. benthamiana.

\begin{tabular}{lllll}
\hline Treatment & $\begin{array}{l}\mathbf{3} \mathbf{d p i}^{\mathbf{a}} \\
\text { inoculation } \\
\text { leaf }\end{array}$ & $\begin{array}{l}\mathbf{3} \mathbf{d p i} \\
\text { systemic } \\
\text { leaf }\end{array}$ & $\begin{array}{l}\mathbf{7} \mathbf{d p i} \\
\text { systemic } \\
\text { leaf }\end{array}$ & $\begin{array}{l}\mathbf{1 0} \mathbf{d p i} \\
\text { systemic } \\
\text { leaf }\end{array}$ \\
\hline LatB & $15 / 15^{\mathrm{b}}$ & $0 / 15$ & $3 / 15$ & $3 / 15$ \\
Oryzalin & $15 / 15$ & $0 / 15$ & $11 / 15$ & $12 / 15$ \\
DMSO & $15 / 15$ & $0 / 15$ & $13 / 15$ & $15 / 15$ \\
\hline
\end{tabular}

a Days post inoculation of RSV in N. benthamiana. ${ }^{b}$ The denominator shows the number of $N$. benthamiana plants used in these treatment; the numerator represents the number of $N$. benthamiana plants with symptom.

expressing NSvc4-eGFP (Compare Figures 4B,G). In marked contrast, the NSvc4 $\Delta$ 106-125-eGFP proteins accumulated as small punctate bodies in the cytoplasm and were not observed at the PD (Figure 4E). These observations indicate that the N-terminal 1125 AA fragment contains a PD localization signal that is sufficient for PD targeting.

\section{LOCALIZATION OF NSvC4 IN SPHERE-LIKE COMPARTMENTS AND CHLOROPLAST}

Image analysis indicated that as well as localizing at the $\mathrm{PD}$, the NSvc4 protein also accumulated in discrete, sphere-like compartments of approximately $4 \mathrm{~mm}$ in diameter in cells (Figure 5). To determine the subcellular localization of these spheres, epidermal cells expressing NSvc4-eGFP were analyzed by confocal microscope. A lambda scan set at $5 \mathrm{~nm}$ intervals between 595 and $755 \mathrm{~nm}$ for analysis of the sphere-like compartments had emission peaks at $500-530 \mathrm{~nm}$ and at $650-700 \mathrm{~nm}$ (Figure 5B). The spectral characteristics of the $650-700 \mathrm{~nm}$ emission peak were similar to the chlorophyll spectrum (maximum at $680 \mathrm{~nm}$ ). So, the fluorescence spectra were collected simultaneously, with one photon multiplier-tube bandwidth set at 500-530 $\mathrm{nm}$ and a second one at $660-700 \mathrm{~nm}$, and the NSvc4-eGFP and chlorophyll 


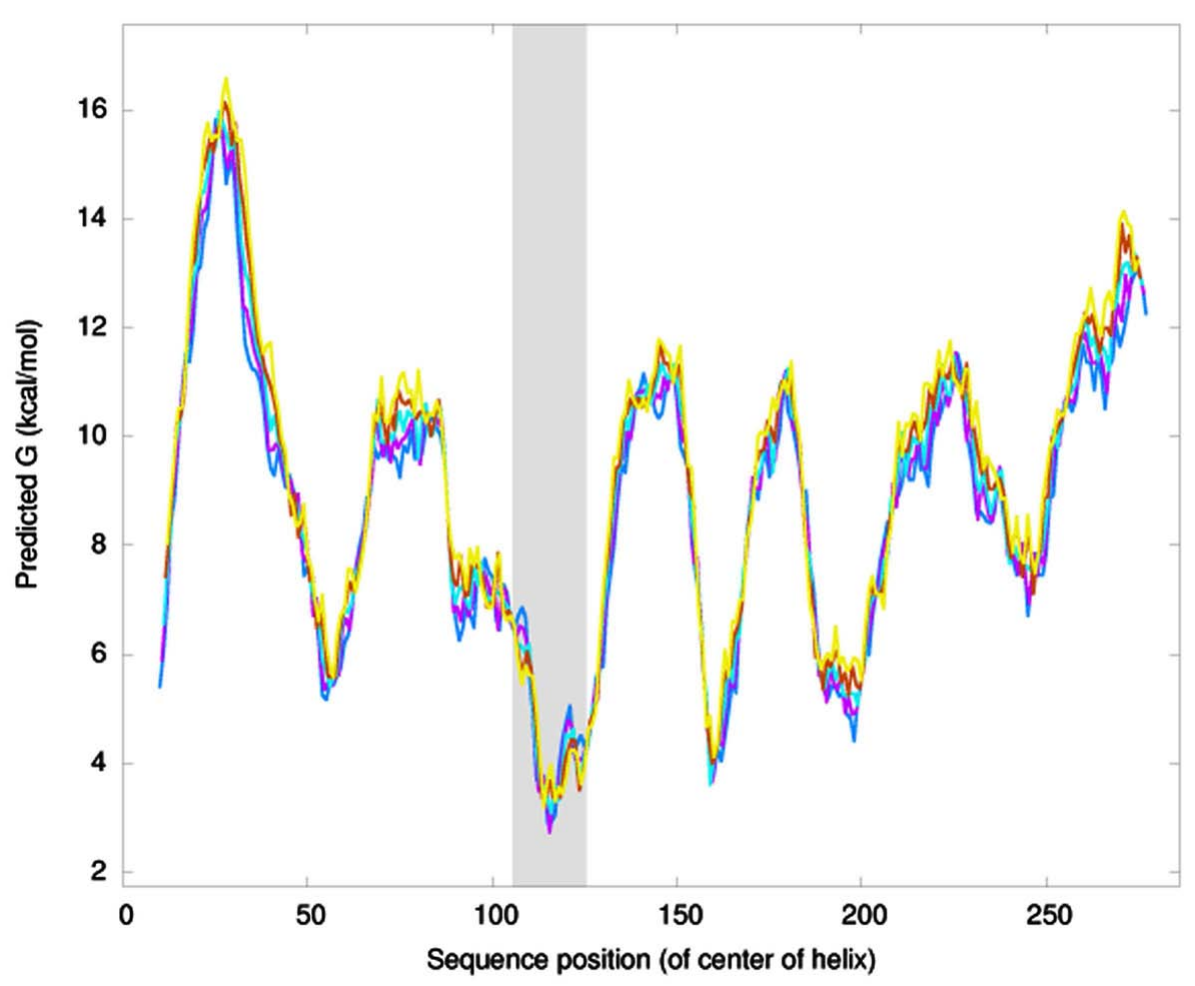

FIGURE 3 | NSVc4 transmembrane domain prediction. Prediction of transmembrane domain was carried out with the $\Delta G$ prediction server (http://dgpred.cbr.su.se/index.php?p=home). The $Y$-axis shows the predicted
$G$ value and the $x$-axis represents the amino acid sequence position. The dark region spanning amino acids $106-125$ is predicted to be a transmembrane domain. autofluorescence was merged to generate yellow fluorescent of the overlapping foci (Figure 5).

To confirm the presence of NSvc4 in the chloroplasts, NSvc4eGFP was expressed in N. benthamiana leaf cells using the PVX vector (pgR107). By 7-8 days post agroinfiltration (dpi), leaves with systemic symptoms were sampled and examined by confocal microscopy. In cells expressing the NSvc4-eGFP, the GFP signal colocalized with the chlorophyll autofluorescence, suggesting that a fraction of the expressed NSvc4-eGFP protein accumulates in the chloroplasts (Figure 6).

To determine which region of NSvc4 is required for chloroplasttargeting, we agroinfiltrated plasmids harboring the wild type or mutant NSvc4-eGFP fusions (Figure 7). The results showed that NSvc4 ${ }_{1-73}$-eGFP accumulated in the sphere-like compartments and in the chloroplasts of the epidermal leaf cells (Figure 7B). However, NSvc4 ${ }_{1-54}$-eGFP and NSvc4 ${ }_{54-73}$-eGFP localized around the nuclei and in the cytoplasm, but were not obvious in the chloroplasts (Figures 7A,E). The remaining NSvc4 mutants localized exclusively in the cytoplasm. These observations suggest that the N-terminal 73 AAs contain a chloroplast-targeting signal.

\section{THE NSvc4 PVX VECTOR INDUCES MORE SEVERE SYMPTOMS IN $\boldsymbol{N}$. BENTHAMIANA THAN PVX}

By 7 days after agroinfiltration of $N$. benthamiana plants for expression of the wtPVX vector (pgR107) or the NSvc4 (pgR-NSvc4), all plants developed systemic symptoms in the upper emerging leaves. Symptoms in plants infiltrated with pgR-NSvc4 were more severe than in plants infiltrated with the wtPVX vector. By 20 dpi, virus symptoms in plants infected with wtPVX vector disappeared, whereas symptoms in the PVX-NSvc4 infected plants remained intense and developed foliar necrosis (Figure 8). Reverse transcription PCR result showed that NSvc4 was accumulated in leaves of the PVX-NSvc4 infected plants in both the early and the late infection stages (data not shown). Examination of thin sections prepared from the PVX or PVX-NSvc4 infected N. benthamiana leaf tissues by electron microscopy revealed major malformations of chloroplast grana and electron lucent bodies beneath the membranes of PVX-NSvc4 infected cells, but similar malformations were not evident in wtPVX infected cells. In addition, proliferations radiating from the chloroplasts into the cytoplasm were observed in the PVX-NSvc4 and RSV rubinoculating infected $N$. benthamiana leaves, but not in the cells infected with wtPVX (Figure 9 and Figure A1 in Appendix).

\section{SYMPTOM DEVELOPMENT IS INDEPENDENT OF NSvc4 CHLOROPLAST LOCALIZATION}

To determine the correlation between NSvc4 chloroplast localization and symptom development, PVX vectors expressing various mutants of NSvc4 were agroinfiltrated individually into $N$. benthamiana leaves. The results demonstrate that $\mathrm{NSvc}_{1-73}$ and NSvc4 $4_{1-106}$ are capable of targeting chloroplasts (Figure 10; Table 2). Interestingly, plants infected with two mutant viruses 

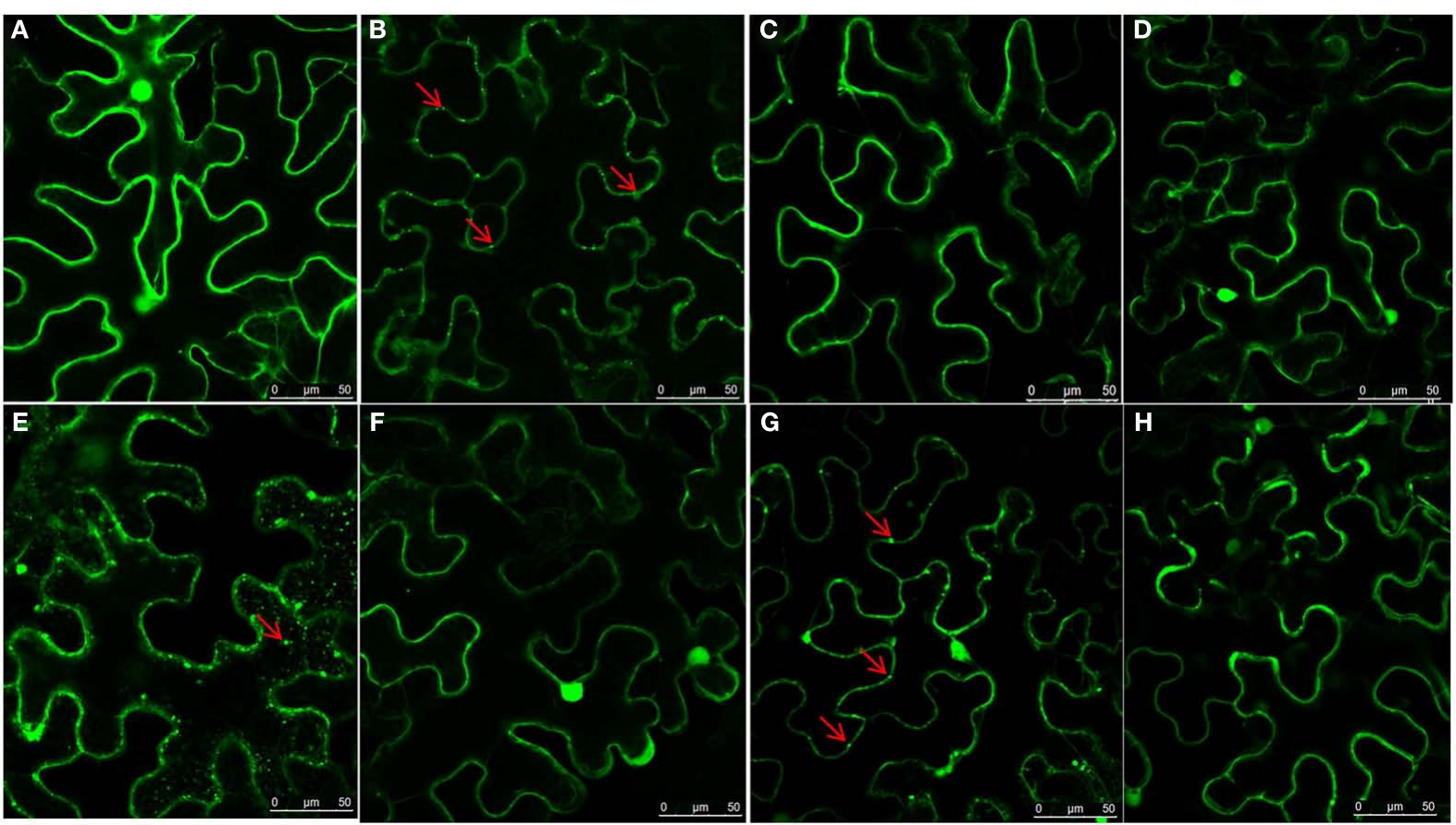

FIGURE 4 | Determination of domains within the NSvc4 that are responsible for PD localization. Tissue was collected from $N$. benthamiana leaves at $48 \mathrm{~h}$ after agroinfiltration with pCHF3-eGFP (A), pCHF3-NSvc4-eGFP (B), pCHF3-NSvc4 ${ }_{1-54}-e G F P(C), p^{-}$pHF3-NSvc4 $_{1-73}-e G F P(D)$,

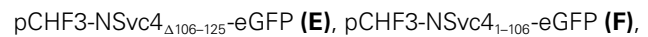
pCHF3-NSvc4 ${ }_{1-125}$-eGFP (G), and pCHF3-NSvc4 ${ }_{125-286}$-eGFP (H). Harvested leaf samples were examined by confocal microscopy. Arrows indicate the localization of NSvc4 and its mutant fusion protein.

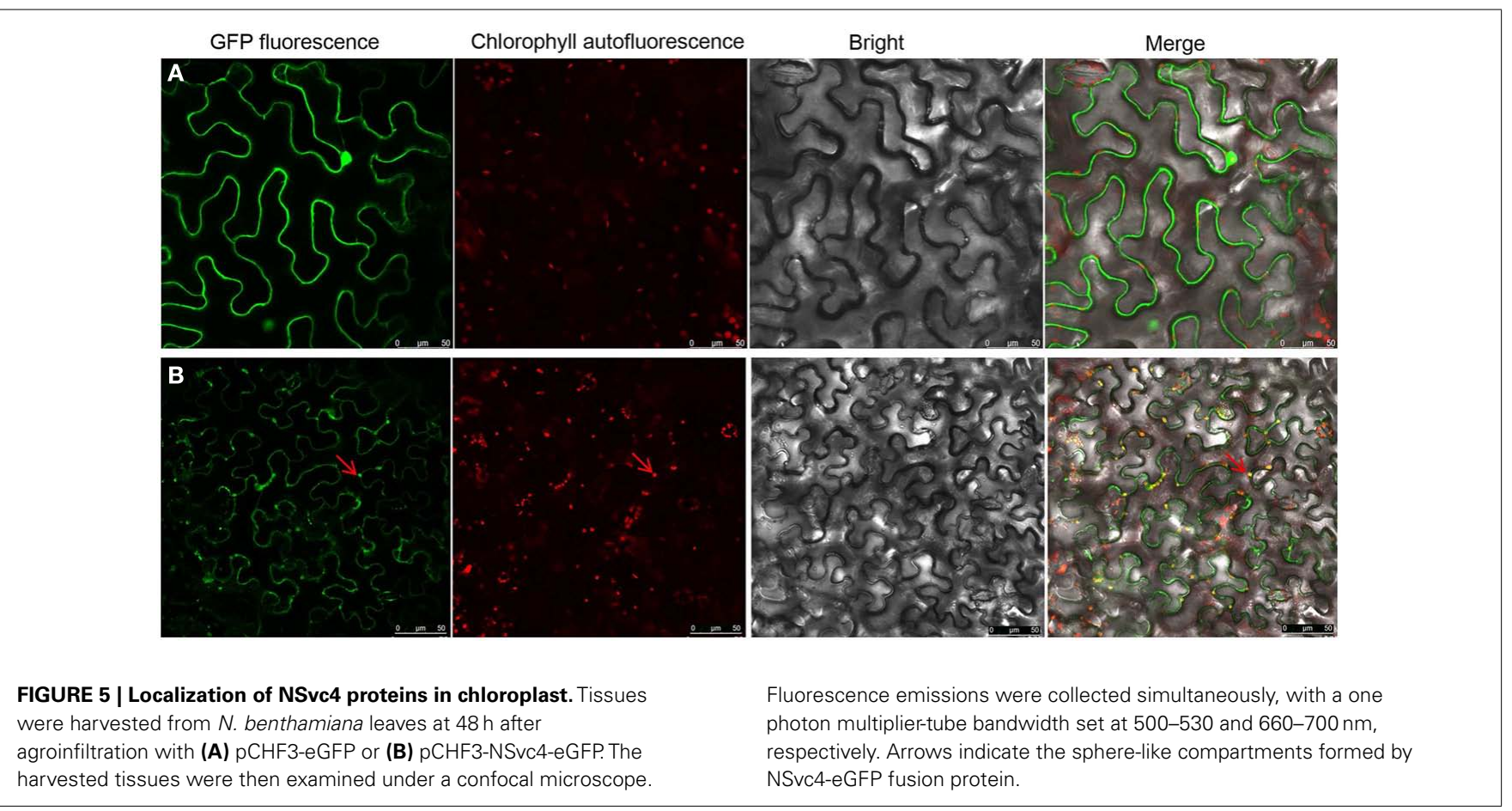

developed phenotypes similar to those caused by the wild wtPVX at $7 \mathrm{dpi}$, and the disease phenotype also recovered by $20 \mathrm{dpi}$. Interestingly, NSvc4 ${ }_{106-286}$ was predicted not to localize to chloroplasts, but, the mutant still elicited a severe symptom phenotype in infiltrated $N$. benthamiana plants that was maintained for up to 20 dpi. These results indicate that NSvc4 chloroplast localization 

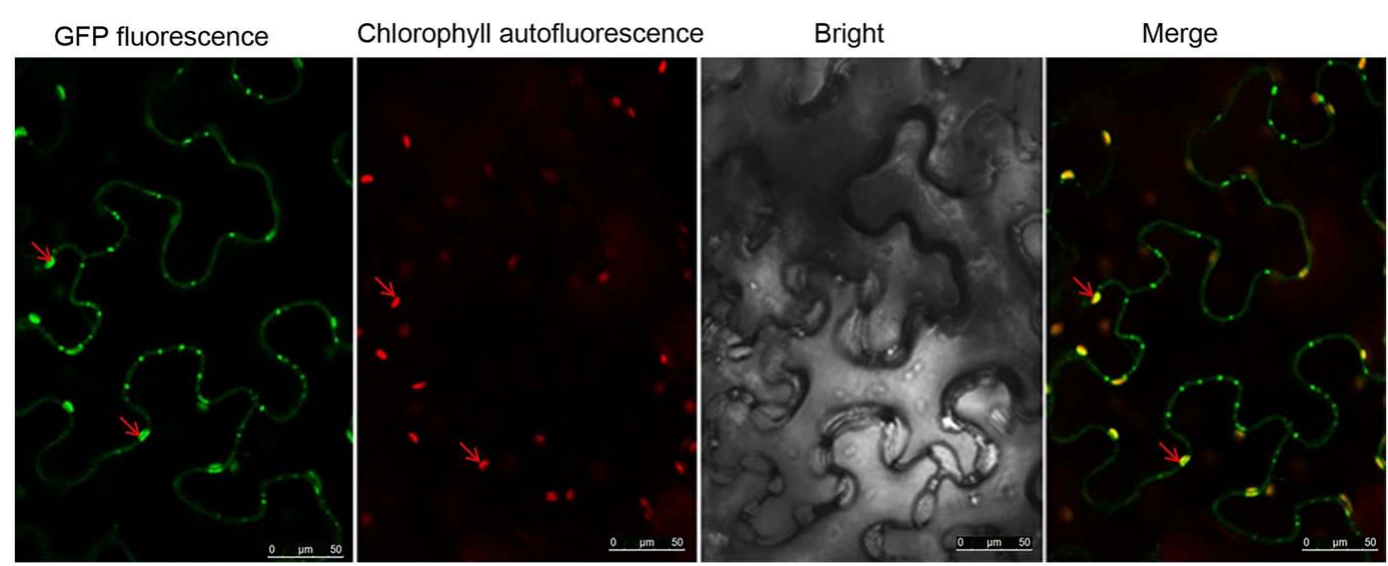

FIGURE 6 | NSvc4-eGFP expressed using the Potato virus X-based vector localized to chloroplasts. At 7 or 8 days post inoculation with pgR-NSvc4-eGFP, systemically infected leaves were sampled and examined by confocal microscope. Arrows indicate the sphere-like compartments formed by NSvc4-eGFP fusion protein.
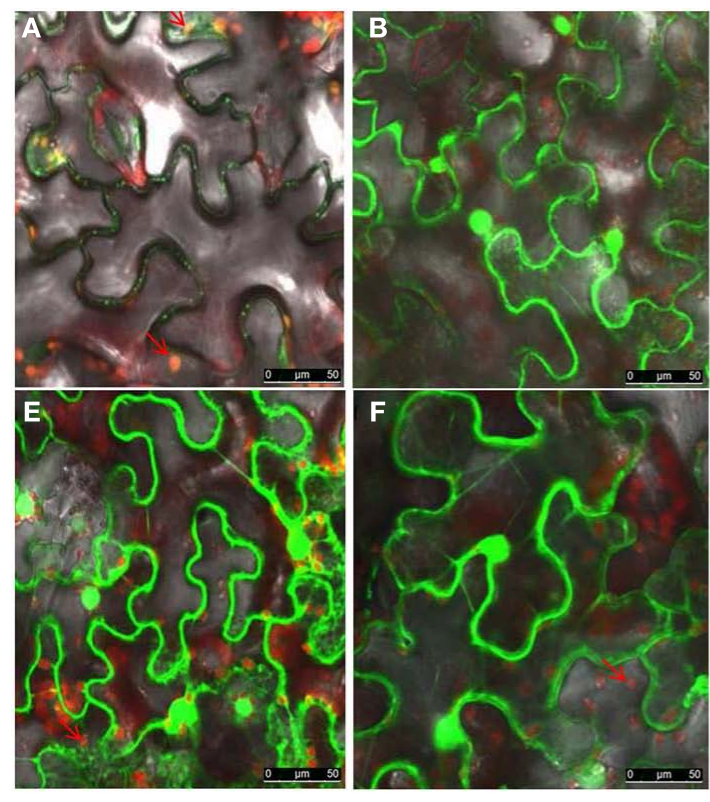
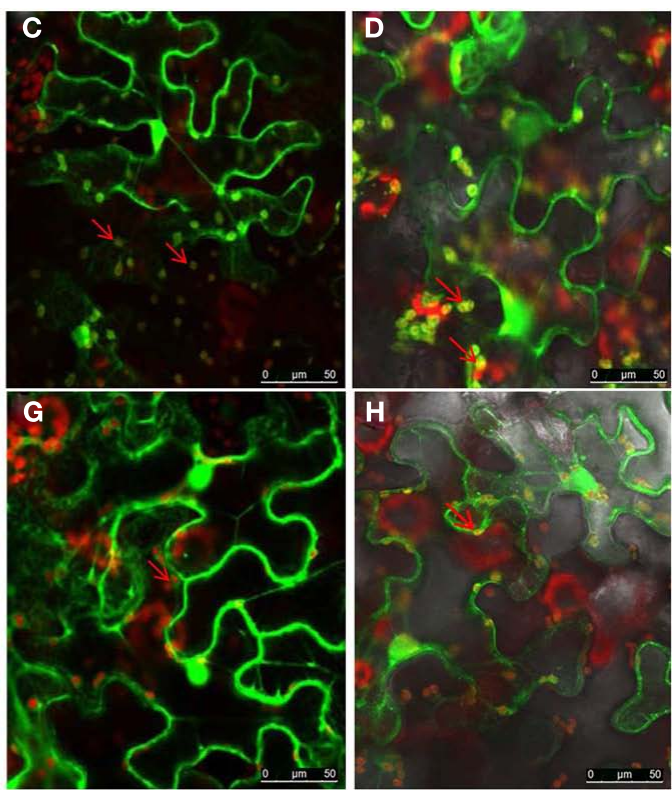

FIGURE 7 |The $\mathbf{N}$-terminus 73 amino acids determine the chloroplast localization of NSvc4. N. benthamiana leaves were agroinfiltrated with

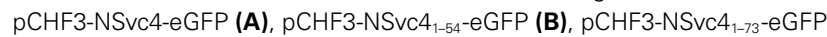
(C), pCHF3-NSvc4 ${ }_{1-125}-e G F P(D), p^{-}$CHF3-NSvc4 $4_{54-73}-e G F P$ (E), pCHF3-NSvc4 ${ }_{125-286}$-eGFP (F), pCHF3-NSvc4 ${ }_{106-125}$-eGFP (G),
pCHF3-NSvc4 ${ }_{\triangle 106-125}-\mathrm{eGFP}(\mathbf{H})$. At $48 \mathrm{~h}$ post agroinfiltration the leaves were sampled and examined under a confocal microscope. The fluorescence emission was collected simultaneously, with one photon multiplier-tube bandwidth set at 500-530 and 660-700 nm, respectively. Arrows indicate the sphere-like compartments and the chloroplast. is dispensable for the exacerbated symptoms. Hence, it is possible that the NSvc4 transmembrane domain has a role in chloroplast malformations, membrane proliferations from the chloroplasts and symptom development.

\section{NSvc4 PROTEIN DID NOT MEDIATE TUBULE FORMATION IN Sf-9 CELLS}

Our earlier research has shown that NSvc4 accumulated at PD in the walls of RSV-infected cells (Xiong et al., 2008). Because the NSm MP of Tomato spotted wilt virus (TSWV) formed tubulelike structures in insect cells (Storms et al., 1995), we decided to investigate the possibility of tubule formation by RSV NSvc4. In these experiments, the TSWV NSm (AcNPV/NSm-GFP) protein elicited numerous tubule-like extensions on Sf-9 cell surface by $36-48 \mathrm{~h}$ post transfection. However, Sf- 9 cells transfected with the RSV NSvc4 protein (AcNPV/NSvc4-GFP), failed to develop similar tubules by $48 \mathrm{hpi}$. In contrast to the free GFP protein (AcNPV/GFP), which was distributed uniformly in the nuclei and in the cytoplasm, the NSvc4-GFP protein (AcNPV/NSvc4-GFP) accumulated as globular structures at the cell periphery and, in this regard, was similar to the localization patterns of NSvc4 in plant 
cells (Figure 11). However, it has been reported in an abstract that the NS2 protein encoded by RSV vRNA 2 can induce tubule-like structures in insect cells (ITMGCM, 1999), but this report has not been verified in a peer reviewed paper. Nevertheless, it is possible that NS2 may interact with NSvc4 to facilitate RSV movement, so in future experiments, we plan to investigate possible roles of NS2

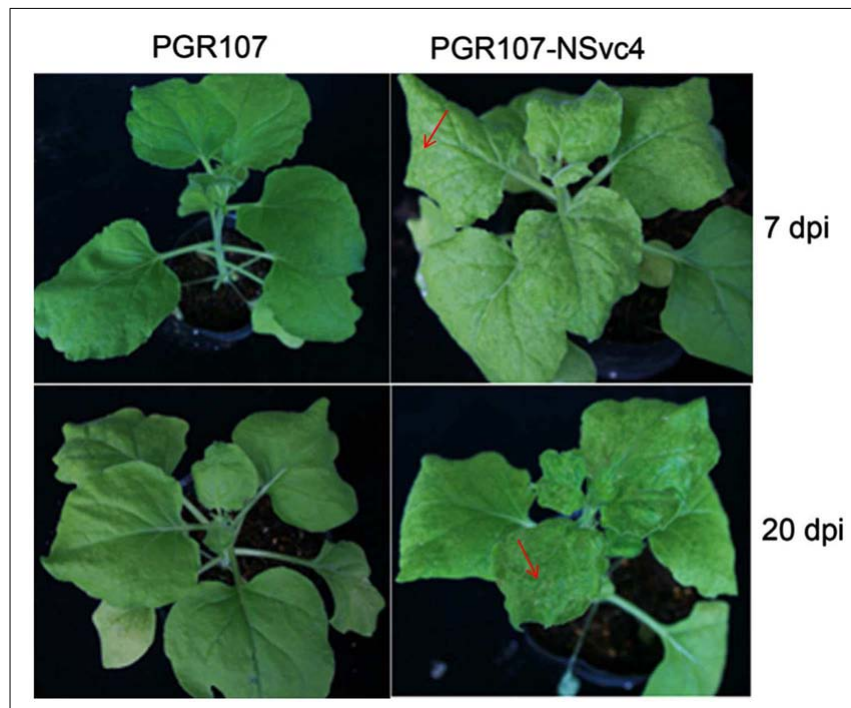

FIGURE 8 | Symptom expression in $\boldsymbol{N}$. benthamiana plants after infection with the wild type PVX vector or the NSVc4 expressing PVX vector. N. benthamiana plants were infiltrated with pgR107 (wild type PVX) or pgR107-NSvc4 (PVX expressing NSvc4) at 7 or at 20 dpi. Allows showed the foliar necrosis in pgR107-NSvc4 infected N. benthamiana. and NSvc4 protein interactions to determine whether they may act together to facilitate RSV cell-to-cell transport.

\section{DISCUSSION}

Our previous studies of RSV NSvc4 have indicated that NSvc4 belongs to the $30 \mathrm{~K}$ MP superfamily, and have shown experimentally that the protein interacts with single-stranded RNA in vitro, traffics to the PD of dicot cells and can move to adjacent cells after

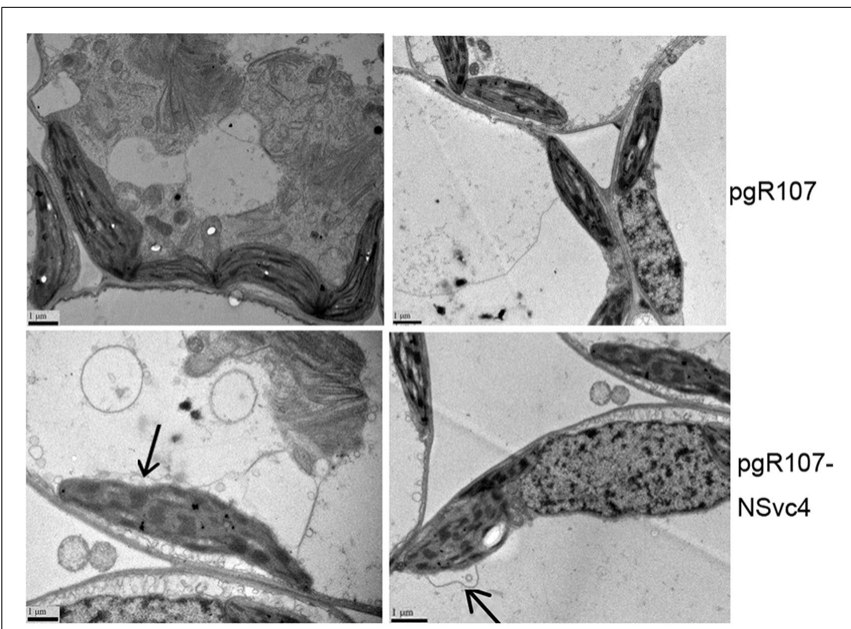

FIGURE 9 | Ultrastructural changes resulting from infection with wild type PVX or PVX-NSvc4. Tissue sections were prepared from the leaves infected with the wild type PVX or PVX expressing NSvc4. Morphological changes in chloroplast were only observed in plants infected with PVX expressing NSvc4. Arrows indicate alterations of chloroplast membrane.

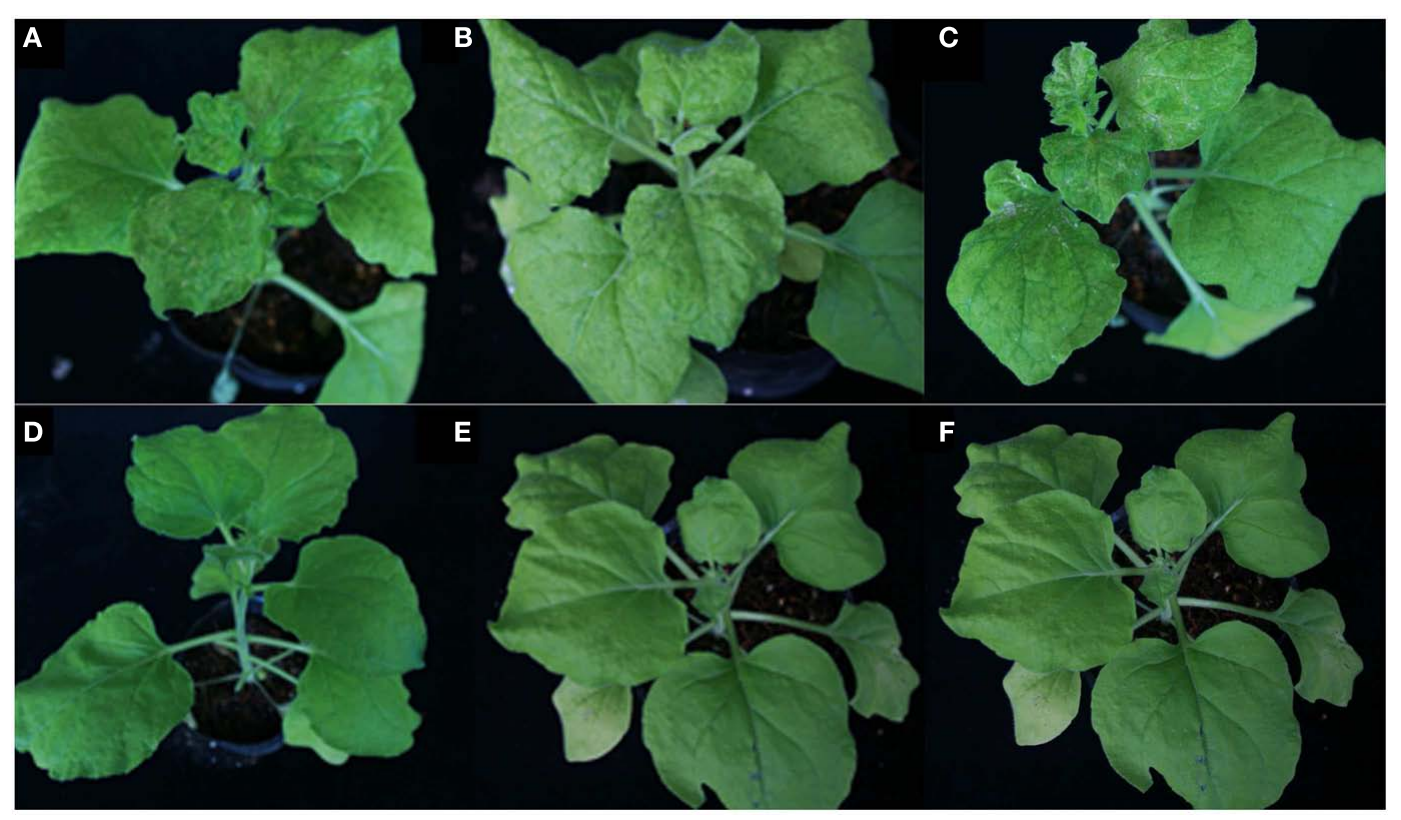

FIGURE 10 | Symptoms elicited by wild type PVX or PVX expressing mutants of NSvc4. N. benthamiana plants were agroinfiltrated with various PVX constructs. Plant infected with PVX-NSvc4 (A), PVX-NSvc4 ${ }_{1-125}$ (B), PVX-NSvc4 ${ }_{125-286}$ (C), PVX (D), PVX-NSvc4 ${ }_{1-53}$ (E), and PVX-NSvc4 ${ }_{1-73}$ (F). Photographs were taken at 20 days post agroinfiltration. 
bombardment (Xiong et al., 2008). Within the $30 \mathrm{~K}$ superfamily, the TMV MP has been studied most intensively and is known to target PD via trafficking on cortical ER and actin cytoskeleton (Wright et al., 2007; Hofmann et al., 2009). A recently report indicates that targeting of NSvc4 to PDs utilizes the actin microfilament pathway and the myosin VIII rather than myosin XI motility system (Yuan et al., 2011). Our results complement and verify this study by demonstrating that actin microfilament dissociation by LatB and Golgi disruption by BFA interfere with PD targeting of the NSvc4-eGFP fusion protein, whereas disruption of microtubules by oryzalin has little effect on PD targeting. Moreover, microfilament disruption but not microtubule disruption inhibits infection of RSV in N. benthamiana, Thus, our combined results clearly suggest that targeting of NSvc4 to PDs depends on a functional ER and actin network.

Many viral MPs within the $30 \mathrm{~K}$ superfamily have a hydrophilic region at their $\mathrm{C}$-termini. Deletion or alanine-scanning mutations within the C-termini of several viral MPs have demonstrated that

Table 2 | Targeting of NSvc4 and its mutants to plasmodesmata and chloroplast and their roles in symptom development.

\begin{tabular}{lccc}
\hline Localization/symptom & Plasmodesmata & Chloroplast & Symptom \\
\hline NSvc4 & + & + & + \\
NSvc4 $_{1-54}$ & - & - & - \\
NSvc4 $_{1-73}$ & - & + & - \\
NSvc4 $_{1-106}$ & - & + & $\mathrm{n}$ \\
NSvc4 $_{1-125}$ & + & + & + \\
NSvc4 $_{125-286}$ & - & - & + \\
NSvc4$_{106-286}$ & - & - & + \\
\hline
\end{tabular}

$n$, The symptom data was not record. this region is dispensable for cell-to-cell movement (Schoelz et al., 2011). We have now extended previous RSV studies through transient expression of wtNSvc4 and NSvc4 mutants in cells, and have determined that NSvc4 differs from the general MP rule because the N-terminal 125 AAs are sufficient to target the truncated fragment to the PD. Furthermore, we have shown that AAs 106-125 contain a predicted transmembrane domain and that deletion of this domain abolishes the PD targeting ability of NSvc4. These results strongly suggest that the 20 deleted residues serve as an integral membrane signal that facilitates insertion into the ER.

Several previous reports have shown that some viral MPs accumulated in chloroplast and thus might have an important role in virus replication, movement, and/or symptom development (Prod'homme et al., 2003; Torrance et al., 2006). For example AltMV TGB3 was shown to be responsible for AltMV movement between cells and contained a novel signal which was required for chloroplast membrane localization. Here we provide definitive evidence that RSV NSvc4 has a chloroplast-targeting signal within its $\mathrm{N}$-terminal 73 residues, and that this signal targets the NSvc4-GFP chloroplast in both the agro-mediated and PVX-based expression systems. We anticipate further studies to elucidate the potential involvement of chloroplast-targeting in the RSV life cycle.

Expression of the NSvc4 through PVX-based vector exacerbated disease symptoms in $N$. benthamiana than the symptoms elicited by PVX alone. Electron microscope observations suggested that disease symptoms correlated with chloroplast malformations and cytoplasmic membrane proliferations in cells. However, expression of mutants of NSvc4 indicated no direct connection between chloroplast localization of NSvc4 and symptom development. We propose that the chloroplast-targeting phenomenon may be involved in RSV replication or other unidentified activities. Considering the chloroplast malformations and membrane proliferations in the PVX-NSvc4 infected N. benthamiana cells, it

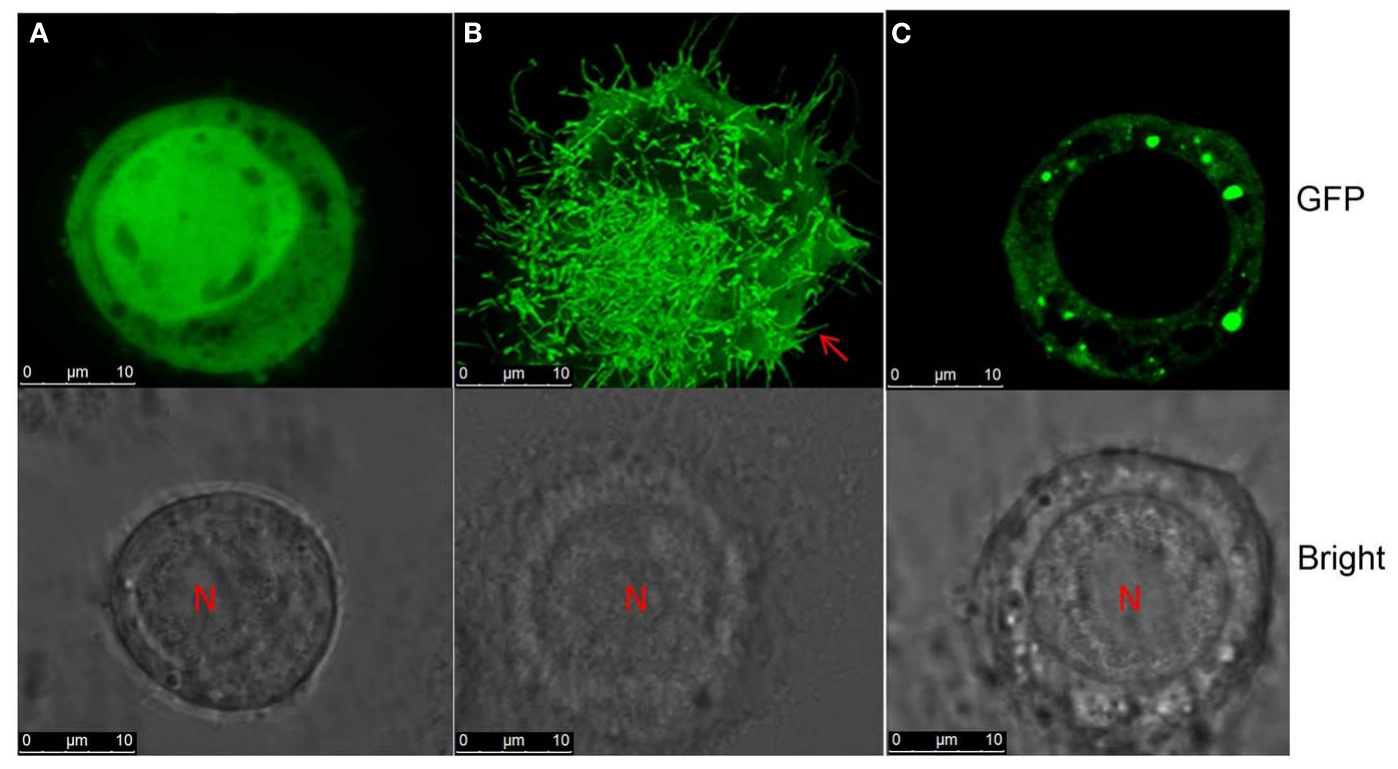

FIGURE 11 |Tubule formation in Sf-9 cells. S. frugiperda 9 cells were transinfected with recombinant baculovirus AcNPV/GFP (A), AcNPV/NSm-GFP (B), or AcNPV/NSvc4-GFP (C). Images were taken at $72 \mathrm{~h}$ post transfection. 
is reasonable to propose that the transmembrane activity of the NSvc4 may play a pivotal role in development of disease symptoms. Because viral MPs modify PD structures and increase PD size exclusion limits, transgenic plants expressing viral MPs often show alterations in plant development. Plant developmental anomalies have also been demonstrated through infection of $N$. benthamiana plants using TMV-based vector expression NSvc4 (Zhang et al., 2012), so the phenomena we have observed are not virus specific. The authors also found that region D17-K33 was recognized as a crucial domain for leaf necrosis response using TMV-based vector expression NSvc4 (Zhang et al., 2012). In our experiment, we also observed foliar necrosis expressed of PVX-NSvc4. More detailed work is needed to determine the regions responsible for formation of foliar necrosis expressed from PVX vector. From these accumulated data, we conclude that RSV NSvc4 is a symptom determinant that affects the host phenotype, but the mechanisms whereby the protein functions in symptom development remain to be elucidated.

One of the major questions unique to RSV movement is the form in which infectious entities might move from initial infection foci to adjacent cells. Because RSV is a negative strand "ambisense" virus, it is obvious that the NC must be involved in intra-cellular transit in order to facilitate nascent transcription and replication in newly invaded plant cells. Similar events also must function during RSV infections of planthopper vectors. Therefore, we carried out preliminary experiments to determine the location of NSvc4 and the TSWV NSm MP in insect cells. Our results show that in contrast to NSm, NSvc4 failed to produce tubule-like structures after plasmid transfection into Sf- 9 cells, but instead formed large foci at the surface of the cells. We previously were unable to detect

\section{REFERENCES}

Aberl, H. J., Rutz, M. L., Karayavuz, M., Frischmuth, S., Wege, C., Hülser, D., et al. (2002). Localizing the movement proteins of abutilon mosaic geminivirus in yeast by subcellular fractionation and freeze-fracture immuno-labelling. Arch. Virol. 147, 103-117.

Ashby, J., Boutant, E., Seemanpillai, M., Groner, A., Sambade, A., Ritzenthaler, C., et al. (2006). Tobacco mosaic virus movement protein functions as a structural microtubule-associated protein. J. Virol. 80, 12433-12433.

Batoko, H., Zheng, H. Q., Hawes, C., and Moore, I. (2000). A rabl GTPase is required for transport between the endoplasmic reticulum and Golgi apparatus and for normal Golgi movement in plants. Plant Cell 12, 2201-2218.

Brandizzi, F., Frangne, N., MarcMartin, S., Hawes, C., Neuhaus, J. M., and Paris, N. (2002). The destination for single-pass membrane proteins is influenced markedly by the length of the hydrophobic domain. Plant Cell 14, 1077-1092.
Brandner, K., Sambade, A., Boutant, E., Didier, P., Mely, Y., Ritzenthaler, C., et al. (2008). Tobacco mosaic virus movement protein interacts with green fluorescent protein-tagged microtubule end-binding protein 1 .

Cao, M., Ye, X., Willie, K., Lin, J., Zhang, X., Redinbaugh, M. G., et al. (2010). The capsid protein of turnip crinkle virus overcomes two separate defense barriers to facilitate systemic movement of the virus in Arabidopsis. J. Virol. 84, 7793-7802.

Du, Z., Xiao, D., Wu, J., Jia, D., Yuan, Z., Liu, Y., et al. (2011). p2 of Rice stripe virus (RSV) interacts with OsSGS3 and is a silencing suppressor. Mol. Plant Pathol. 12, 808-814.

Falk, B. W., and Tsai, J. H. (1998). Biolin the genus tenuivirus. Annu. Rev. Phytopathol. 36, 139-163.

Green, T. J., Rowse, M., Tsao, J., Kang, J., Ge, P., Zhou, Z. H., et al. (2011). Access to RNA encapsidated in the nucleocapsid of vesicular stomatitis virus. J. Virol. 85, 2714-2722.

Harries, P. A., Palanichelvam, K., Yu, W., Schoelz, J. E., and Nelson, R. Plant Physiol. 147, 611-623. ogy and molecular biology of viruses

NSvc4 binding to the RSV NC protein, but have shown that NSvc4 exhibits non-specific RNA binding in gel shift assays. These results suggest that NSvc4 may be able to access RNA encapsidated in the $\mathrm{NC}$, and such a mechanism is compatible with recent experimental data for Vesicular stomatitis virus (VSV), the most intensively studied negative strand virus (Green et al., 2011). Interestingly, the VSV NC (N) protein is thought to undergo conformational changes to permit access by the polymerase protein during transcription and replication. Moreover, the matrix protein of negative strand viruses has mechanisms to discriminate genomic NCs from antigenomic NCs during morphogenesis, and these likely are RNA sequence specific. Therefore, we posit that NSvc4 specifically recognizes RNA in RSV gNCs and ferries these complexes to the cell wall and then enlarges the PD complexes sufficiently to facilitate NC transit to adjacent cells. Although, different mechanisms, possibly cell fusion, may be involved in systemic spread in infected planthoppers, we envision that NSvc4:NC associations with NCs likely function during insect infection processes. Therefore, we are planning further investigations to elucidate the complicated mechanisms whereby RSV moves between plant and insect cells.

\section{ACKNOWLEDGMENTS}

This research was supported by the Hi-tech Research and Development Program of China (2012AA101505) and the National Natural Science Foundation of China (Grant No. 30870110). We thank Prof. Chuanxi Zhang in Zhejiang University for supply of pFastBacHT-eGFPT and E. coli DH1OBac, Qianli An for supplying Man1-RFP plasmids. We apologize to colleagues whose articles were not cited because of space limitations. TSWV NSm was kindly provided by Prof. Jian Hong in Zhejiang University.

S. (2009a). The cauliflower mosaic virus protein $\mathrm{P} 6$ forms motile inclusions that traffic along actin microfilaments and stabilize microtubules. Plant Physiol. 149, 1005-1016.

Harries, P. A., Park, J. W., Sasaki, N., Ballard, K. D., Maule, A. J., and Nelson, R. S. (2009b). Differing requirements for actin and myosin by plant viruses for sustained intercellular movement. Proc. Natl. Acad. Sci. U.S.A. 106, 17594-17599.

Harries, P. A., Schoelz, J. E., and Nelson, R. S. (2010). Intracellular transport of viruses and their components: utilizing the cytoskeleton and membrane highways. Mol. Plant Microbe Interact. 23, 1381-1393.

Hessa, T., Meindl-Beinker, N. M., Bernsel, A., Kim, H., Sato, Y., LerchBader, M., et al. (2007). Molecular code for transmembrane-helix recognition by the Sec61 translocon. Nature 450, 1026-1030.

Hibino, H. (1996). Biology and epidemiology of rice viruses. Annu. Rev. Phytopathol. 34, 249-274.

Hofmann, C., Niehl, A., Sambade, A., Steinmetz, A., and Heinlein, M. (2009). Inhibition of tobacco mosaic virus movement by expression of an actin-binding protein. Plant Physiol. 149, 1810-1823.

Huang, Z., Han, Y., and Howell, S. H. (2000). Formation of surface tubules and fluorescent foci in Arabidopsis thaliana protoplasts expressing a fusion between the green fluorescent protein and the cauliflower mosaic virus movement protein. Virology 271, 58-64.

ITMGCM. (1999). Expression of rice stripe virus $228 \mathrm{k}$ protein in insect cell. Annu. Phytopathol. Soc. Jpn. 65, 337.

Laporte, C., Vetter, G., Loudes, A. M., Robinson, D. G., Hillmer, S., Stussi-Garaud, C., et al. (2003). Involvement of the secretory pathway and the cytoskeleton in intracellular targeting and tubule assembly of grapevine fanleaf virus movement protein in tobacco BY-2 cells. Plant Cell 15, 2058-2075.

Li, S., Xiong, R. Y., Wang, X. F., and Zhou, Y. J. (2011). Five proteins of Laodelphax striatellus are potentially involved in the interactions between rice stripe virus and vector. PLoS ONE 6:e26585. doi:10.1371/journal.pone.0026585. 
Lim, H. S., Bragg, J. N., Ganesan, U., Ruzin, S., Schichnes, D., Lee, M. Y., et al. (2009). Subcellular localization of the barley stripe mosaic virus triple gene block proteins. J. Virol. 83, 9432-9448.

Lim, H. S., Vaira, A. M., Bae, H., Bragg, J. N., Ruzin, S. E., Bauchan, G. R., et al. (2010). Mutation of a chloroplasttargeting signal in alternanthera mosaic virus TGB3 impairs cell-tocell movement and eliminates longdistance virus movement. J. Gen. Virol. 91, 2102-2115.

Paape, M., Solovyev, A. G., Erokhina, T. N., Minina, E. A., Schepetilnikov, M. V., Lesemann, D. E., et al. (2006). At-4/1, an interactor of the tomato spotted wilt virus movement protein, belongs to a new family of plant proteins capable of directed intra- and intercellular trafficking. Mol. Plant Microbe Interact. 19, 874-883.

Pouwels, J., Van der Krogt, G. N. M., Van Lent, J., Bisseling, T., and Wellink, J. (2002). The cytoskeleton and the secretory pathway are not involved in targeting the cowpea mosaic virus movement protein to the cell periphery. Virology 297, 48-56.

Prod'homme, D., Jakubiec, A., Tournier, V., Drugeon, G., and Jupin, I. (2003). Targeting of the turnip yellow mosaic virus $66 \mathrm{~K}$ replication protein to the chloroplast envelope is mediated by the $140 \mathrm{~K}$ protein. J. Virol. 77, 9124-9135.

Qu, F., Ren, T., and Morris, T. J. (2003). The coat protein of turnip crinkle virus suppresses posttranscriptional gene silencing at an early initiation step. J. Virol. 77, 511-522.

Schoelz, J. E., Harries, P. A., and Nelson, R. S. (2011). Intracellular transport of plant viruses: finding the door out of the cell. Mol. Plant 4, 813-831.

Scholthof, H. B. (2005). Plant virus transport: motions of functional equivalence. Trends Plant Sci. 10, 376-382.
Shen, Q., Liu, Z., Song, F., Xie, Q. Hanley-Bowdoin, L., and Zhou, X. (2011). Tomato SISnRK1 protein interacts with and phosphorylates betaC1, a pathogenesis protein encoded by a geminivirus beta-satellite. Plant Physiol. 157, 1394-1406.

Shimizu, T., Yoshii, A., Sakurai, K., Hamada, K., Yamaji, Y., Suzuki, M., et al. (2009). Identification of a novel tobacco DnaJ-like protein that interacts with the movement protein of tobacco mosaic virus. Arch. Virol. 154, 959-967.

Snider, C., Jayasinghe, S., Hristova, K., and White, S. H. (2009). MPEx: a tool for exploring membrane proteins. Protein Sci. 18, 2624-2628.

Storms, M. M., Kormelink, R., Peters, D., Van Lent, J. W., and Goldbach, R. W. (1995). The nonstructural NSm protein of tomato spotted wilt virus induces tubular structures in plant and insect cells. Virology 214, 485-493.

Tilsner, J., Linnik, O., Wright, K. M., Bell, K., Roberts, A. G., Lacomme, C., et al. (2012). The TGB1 movement protein of potato virus $\mathrm{X}$ reorganizes actin and endomembranes into the X-body, a viral replication factory. Plant Physiol. 158, 1359-1370.

Toriyama, S. (1986). Rice stripe virus: prototype of a new group of viruses that replicate in plants and insects. Microbiol. Sci. 3, 347-351.

Torrance, L., Cowan, G. H., Gillespie, T., Ziegler, A., and Lacomme, C. (2006). Barley stripe mosaic virusencoded proteins triple-gene block 2 and gammab localize to chloroplasts in virus-infected monocot and dicot plants, revealing hitherto-unknown roles in virus replication. J. Gen. Virol. 87, 2403-2411.

Tse, Y. C., Lo, S. W., Hillmer, S., Dupree, P., and Jiang, L. (2006). Dynamic response of prevacuolar compartments to brefeldin a in plant cells. Plant Physiol. 142, 1442-1459.
Verchot-Lubicz, J., Torrance, L., Solovyev, A. G., Morozov, S. Y., Jackson, A. O., and Gilmer, D. (2010). Varied movement strategies employed by triple gene block-encoding viruses. Mol. Plant Microbe Interact. 23, 1231-1247.

Wei, T., Huang, T. S., McNeil, J., Laliberte, J. F., Hong, J., Nelson, R. S., et al. (2010a). Sequential recruitment of the endoplasmic reticulum and chloroplasts for plant potyvirus replication. J. Virol. 84, 799-809.

Wei, T. Y., Zhang, C. W., Hong, J. A., Xiong, R. Y., Kasschau, K. D., Zhou, X., et al. (2010b). Formation of complexes at plasmodesmata for potyvirus intercellular movement is mediated by the viral protein $\mathrm{P} 3 \mathrm{~N}$ PIPO. PLoS Pathog. 6:e1000962. doi:10.1371/journal.ppat.1000962.

Wei, T. Y., Yang, J. G., Liao, F. R., Gao F. L., Lu, L. M., Zhang, X.-T., et al. (2009). Genetic diversity and population structure of rice stripe virus in China. J. Gen. Virol. 90, 1025-1034.

Wright, K. M., Wood, N. T., Roberts, A. G., Chapman, S., Boevink, P., Mackenzie, K. M., et al. (2007). Targeting of TMV movement protein to plasmodesmata requires the actin/ER network: evidence from FRAP. Traffic 8, 21-31.

Xiong, R., Wu, J., Zhou, Y., and Zhou, $X$. (2008). Identification of a movement protein of the tenuivirus rice stripe virus. J. Virol. 82, 12304-12311.

Xiong, R., Wu, J., Zhou, Y., and Zhou, X. (2009). Characterization and subcellular localization of an RNA silencing suppressor encoded by rice stripe tenuivirus. Virology 387 29-40.

Yuan, Z., Chen, H., Chen, Q., Omura T., Xie, L., Wu, Z., et al. (2011). The early secretory pathway and an actin-myosin VIII motility system are required for plasmodesmatal localization of the NSvc4 protein of rice stripe virus. Virus Res. 159, 62-68.
Zhang, C., Pei, X., Wang, Z., Jia, S., Guo, S., Zhang, Y., et al. (2012). The rice stripe virus pc4 functions in movement and foliar necrosis expression in Nicotiana benthamiana. Virology 425, 113-121.

Zhang, S. C., Ghosh, R., and Jeske, H. (2002). Subcellular targeting domains of abutilon mosaic geminivirus movement protein BC1. Arch. Virol. 147, 2349-2363.

Zhang, S. C., Wege, C., and Jeske, H. (2001). Movement proteins (BC1 and BV1) of abutilon mosaic geminivirus are cotransported in and between cells of sink but not of source leaves as detected by green fluorescent protein tagging. Virology 290, 249-260.

Zhao, S. L., Dai, X. J., Liang, J. S., and Liang, C. Y. (2012). Surface display of rice stripe virus NSvc2 and analysis of its membrane fusion activity. Virol. Sin. 27, 100-108.

Conflict of Interest Statement: The authors declare that the research was conducted in the absence of any commercial or financial relationships that could be construed as a potential conflict of interest.

Received: 09 October 2012; accepted: 19 November 2012; published online: 07 December 2012.

Citation: Xu Y and Zhou X (2012) Role of rice stripe virus NSvc4 in cell-to-cell movement and symptom development in Nicotiana benthamiana. Front. Plant Sci. 3:269. doi: 10.3389/fpls.2012.00269

This article was submitted to Frontiers in Plant-Microbe Interaction, a specialty of Frontiers in Plant Science.

Copyright (C) $2012 \mathrm{Xu}$ and Zhou. This is an open-access article distributed under the terms of the Creative Commons Attribution License, which permits use, distribution and reproduction in other forums, provided the original authors and source are credited and subject to any copyright notices concerning any third-party graphics etc. 


\section{APPENDIX}

Table A1 | Primers used in our experiments.

\begin{tabular}{ll}
\hline MP(Kpn1)-F & 5'-ggggtaccATGGCTTTGTCTCGACTTTTG-3' \\
MP(BamH1)-R & 5'-CGGGATCCcatgatgacagaaacttcag-3' \\
MP(BamH1)-R54 & 5'-GGATCCtgtggcagcttggtcaatc-3' \\
MP(BamH1)-R73 & 5'-GGATCCATCATACTTGTTCACCTTGACAT-3' \\
MP(BamH1)-R106 & 5'-CGGGATCCatgggtgagaggttgatg-3' \\
MP(kpn1)-F125 & 5'-gggtaccATGagtggaataactaccctcc-3' \\
MP(Kpn1)-F106 & 5'-gggtaccATGtatccattcttagagtggc-3' \\
MP(BamH1)-R125 & 5'-CGGGATCCagctctacccttgattcct-3' \\
MP-Fol & 5'-cctctcacccatagtggaataactaccctc-3' \\
MP-Rol & 5'-agttattccactatgggtgagaggttgatg-3' \\
eGFP(Sal1)-R & 5'-GTCGACTTACTTGTACAGCTCGTCCAT-3' \\
MP(sal1)-R54 & 5'-GTCGACCTAtgtggcagcttggtcaatc-3' \\
MP(sal1)-R73 & 5'-GTCGACCTAATCATACTTGTTCACCTTGACAT-3' \\
MP(sal1)-R125 & 5'-GTCGACCTAagctctacccttgattcct-3' \\
MP(Cla1)-F125 & 5'-CCATCGATATGagtggaataactaccctcc-3' \\
MP(Cla1)-F & 5'-ATCGATATGGCTTTGTCTCGACTTTT-3' \\
MP(sal1)-R & 5'-GTCGACCTACATGATGACAGAAACTTC-3' \\
MP-(BamH1)-F & 5'-GGATCCATGGCTTTGTCTCGACTTTT-3' \\
MP-(Sal1)-R & 5'-GTCGACCTACATGATGACAGAAACTTC-3' \\
&
\end{tabular}

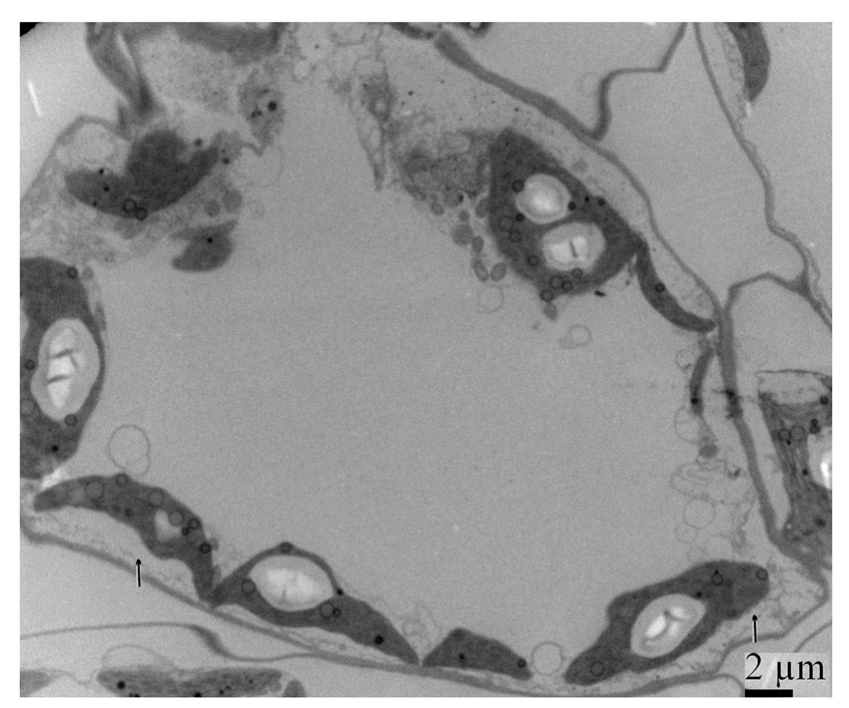

FIGURE A1 | Ultrastructural structures resulting from 20 days post rub-inoculating $N$. benthamiana leaves with crude extracts from RSV-infected $\boldsymbol{O}$. sativa leaves. Allows showed that membrane proliferations radiating from the chloroplasts into the cytoplasm. 